\title{
Individualización y prevención social del delito en Argentina: la reactualización de las "clases peligrosas"
}

\author{
Emilio J. Ayos \\ Universidad de Buenos Aires (UBA)
}

Individualización y prevención social del delito en Argentina: la reactualización de las "clases peligrosas"

Resumen: El objetivo del artículo es presentar algunos avances de investigación en torno a lo que ha sido conceptualizado como procesos de individualización en la prevención "social" del delito, a partir de un trabajo que delimitó como su objeto de estudio las formas en las que se construyó la pobreza y el delito en la confluencia de los Programas Comunidades Vulnerables y de Empleo Comunitario. De esta manera, a partir de un enfoque metodológico cualitativo que combinó el análisis de documentos de gestión y de entrevistas en profundidad a sus agentes implementadores, el artículo describirá la tendencia hacia la construcción de dicha intervención como una intervención sobre la individualidad, produciendo un descentramiento del impacto en las condiciones de vida como operación preventiva de ilegalismos, e instando una particular vinculación entre lo delictivo y la pobreza, y en términos más generales, entre lo delictivo y las condiciones de vida.

Palabras claves: pobreza, delito, clases peligrosas, política social, política criminal.

Individualização e prevenção social do delito na Argentina: a readaptação das "classes perigosas" Resumo: O objetivo deste artigo é apresentar alguns avanços da pesquisa em torno do que se conceitua como processos de individualização na prevenção "social" do delito, a partir de um trabalho que delimitou como seu objeto de estudo as formas em que se construiu a pobreza e o delito, na confluência dos Programas Comunidades Vulneráveis e de Emprego Comunitário. Desta maneira, a partir de um enfoque metodológico qualitativo, que combinou a análise de documentos de gestão e de entrevistas em profundidade a seus agentes implementadores, o artigo descreverá a tendência em direção à construção de tal intervenção sobre a individualidade, produzindo um descentramento do impacto nas condições de vida como operação preventiva da infração, e instando uma vinculação particular entre o delitivo e a pobreza, e, em termos mais gerais, entre o delitivo e as condições de vida.

Palavras-chave: pobreza, delito, classes perigosas, política social, política criminal.

\section{Individualization and Social Prevention of Crime in Argentina: the Revival of the "Dangerous} Classes"

Abstract: The purpose of this article is to present some research advances about what has been conceptualized as processes of individualization in the "social" prevention of crime, based on a study of the ways in which poverty and crime are constructed in the confluence of Vulnerable Community Programs and Community Employment. In this way, based on a qualitative methodological focus that combines analysis of administrative documents and in-depth interviews with implementing agents, the article describes the tendency towards the construction of individualistic interventions, shifting the focus on changing living conditions as a method to prevent crime, and establishing a particular link between crime and poverty and in more general terms, between crime and living conditions.

Key words: poverty, crime, dangerous classes, social policy, criminal policy. 


\section{Introducción}

Este artículo presenta algunos resultados de una investigación que se enfoca en los procesos de imbricación y solapamiento de las definiciones ligadas a las nociones de pobreza y delito y las estrategias de intervención asociadas a las mismas, en los campos de la política social y la política criminal argentina entre los años 2003 y 2008. Específicamente a partir de la confluencia de dos intervenciones estatales concretas: el Programa Comunidades Vulnerables (PCV) y el Programa de Empleo Comunitario (PEC). El PEC es creado por el Ministerio de Trabajo, Empleo y Seguridad Social (MTEySS) en el año 2003, como cristalización de experiencias originadas en el interior del Programa de Emergencia Laboral (PEL) iniciado en 1999 y en el marco de la aparición del Plan Jefas y Jefes de Hogar Desocupados en el año 2002 ${ }^{1}$. En el contexto de la declaración de la emergencia social, económica, administrativa, financiera y cambiaria por parte del gobierno nacional, el MTEySS decide abrir la incorporación al PEL a sectores que quedaban por fuera de la delimitación de la población-objetivo del Plan Jefas y Jefes de Hogar Desocupados, especialmente los jóvenes. En ese sentido, se dispone un monto del beneficio similar al programa anterior, y como contraprestación la realización de actividades "comunitarias" de carácter mensual, para 300 mil beneficiarios. A partir de esta experiencia anterior dentro del PEL, nace en enero del 2003 el PEC, con el objetivo de "promover la participación de trabajadores desocupados en situación de vulnerabilidad social en proyectos que mejoren su empleabilidad y faciliten su inserción laboral".

El PCV surge en el año 2001 como parte del naciente Plan Nacional de Prevención del Delito (PNPD) dependiente del Ministerio de Justicia y Derechos Humanos de la Nación, incorporando y coordinando también programas locales anteriores (por ejemplo las experiencias de la Ciudad de Buenos Aires). Este se aplicó hasta el mes de abril de 2008 en diferentes puntos de la Ciudad de Buenos Aires, del Gran Buenos Aires, además de hacerlo en otras provincias. Su implementación fue "focalizada en barrios de alta vulnerabilidad social", abocada a dos ejes de acción interrelacionados: a) la prevención de la violencia social y b) la prevención del delito.

La formulación original del programa se organizaba a partir de tres áreas: la jurídica, que disponía de orientación y derivación jurídica en materia penal y familiar; el área vincular, que preveía acciones para contrarrestar deficiencias en el funcionamiento de las relaciones vinculares originadas en las distintas situaciones de vulnerabilidad, como entre otras, actividades culturales, artísticas, recreativas y deportivas, talleres de prevención de la violencia y el uso indebido de drogas; y por último, el área de microemprendimientos, que intentaba estimular su organización, entendiéndolos como una forma de reducir las situaciones de "riesgo". La población “objetivo" fue construida en torno a la noción de "jóvenes en conflicto con la ley penal".

En cuanto a la relación entre los programas mencionados, hemos encontramos una vinculación empírica del PCV, desde su lanzamiento, con los planes asistenciales administrados por el Ministerio de Trabajo, Empleo y Seguridad Social, y específicamente desde el año 2003, con el PEC; este punto marca nuestro recorte temporal. Y observamos que el otorgamiento de estos planes es un factor fundamental en las estrategias de prevención social del delito implementadas por el PCV, puesto que el beneficio correspondiente al PEC es el único que se distribuyó a la "población-objetivo" como retribución a las actividades que establece el programa.

Para completar esta introducción haremos referencia primero a la estrategia metodológica utilizada. Luego se hará una reconstrucción históricoconceptual de nuestro problema general de investigación. Posteriormente presentarán los resultados en torno a lo que hemos conceptualizado como procesos de individualización que se expresan en la articulación del PCV y el PEC. Por último, concluiremos con unas reflexiones finales acerca de estos resultados presentados.

\section{Metodología}

Para la realización de los objetivos propuestos en la presente investigación se adoptó un enfoque metodológico cualitativo. Siguiendo los lineamientos y procedimientos propuestos por la teoría fundada en los datos (Grounded Theory) (GLASER; STRAUSS, 1967; SONEIRA, 2004), se seleccionaron para la realización del trabajo de campo la implementación de los Programas "Comunidades Vulnerables" y de "Empleo Comunitario" en dos municipios dentro del área metropolitana de la Ciudad de Buenos Aires. La estrategia de investigación combinó el uso de fuentes primarias y secundarias. En cuanto a las primeras, se realizaron entrevistas abiertas fundamentalmente a tres grupos de individuos: a las autoridades nacionales de dichas intervenciones, las autoridades locales de los mismos, y los "operadores comunitarios".

En cuanto a las fuentes secundarias, se conformó un corpus con documentos provenientes de la Dirección Nacional de Política Criminal y del Ministerio de Trabajo, referido a los programas bajo estudio. Por último se expresa que el proyecto de investigación del cual surge el presente artículo fue evaluado satisfactoriamente en sus aspectos técni- 
cos como éticos por el Consejo Nacional de Investigaciones Científicas y Técnicas, del cual soy Becario Doctoral. Así mismo, se deja constancia que fue solicitado el libre consentimiento de los sujetos entrevistados para esta investigación. Se ha asegurado la confidencialidad y privacidad de sus testimonios, garantizando la no utilización de informaciones en perjuicio de los que participaron de la investigación, y observándose las normas legales y éticas para las investigaciones que involucran seres humanos.

\section{Cuestión social y clases peligrosas: pobreza, peligrosidad y delito}

Las viejas poor laws isabelinas se caracterizaron por mantener una relación ambigua con la figura del "indigente válido". Castel (1997) las integra dentro de lo que conceptualiza como sistemas socialesasistenciales, los cuales aparecen en Europa cuando comienzan a debilitarse las redes de integración primaria características de sociedades donde no se configura un ámbito diferenciado de "lo social"; estos sistemas implementaron lenta pero progresivamente prácticas especializadas de asistencia, con niveles crecientes de tecnificación en la intervención, y que estimulan formas de sociabilidad secundaria. Por otro lado, establecieron dos requisitos generales para la asistencia: el de pertenencia comunitaria y el de la "ineptitud para el trabajo". Pero si bien estos principios aparecieron con regularidad en la letra de las leyes, en la práctica, el segundo de ellos mostró una situación más compleja.

"La dañosa ambigüedad de la palabra pobre", cita Himmelfarb (1983) del Poor Law Report de 1834. La frase hace referencia crítica a la indiferenciación propia de las Leyes de Pobres que imperaban en Inglaterra hasta el primer tercio del siglo 19, las cuales no hacían una distinción cabal especialmente en su aplicación - entre el pobre "válido" y el incapacitado para el trabajo. Tanto las Leyes de Pobres de 1536 hasta 1601 y el estatuto de artífices de 1563, como la Ley de Asentamientos de 1662, que en conjunto Polanyi (1992) califica como un verdadero código laboral, configuraban una serie de instrumentos fundamentados en los principios del paternalismo y la regulación inherentes a las relaciones tradicionales de dependencia y vasallaje. Salvo excepcionales períodos donde se ampliaba la unidad administrativa, la parroquia era el agente encargado de la implementación de estas intervenciones, quedando bajo su cargo la distribución de la asistencia. Es en el marco de estas relaciones de proximidad comunal, atravesados por los principios de interdependencia tradicional, que los criterios de discriminación en torno a la capacidad de trabajo se vuelven laxos o débiles.
Ahora bien, un sistema de este tipo se corresponde con las relaciones sociales que se despliegan en estas economías precapitalistas, donde todavía no se ha conformado un mercado de trabajo a nivel nacional. Precisamente, en el momento en que la dinámica del desarrollo económico llegó a los albores de la Revolución Industrial, y un mercado de trabajo libre de las regulaciones tradicionales apareció como una necesidad, estos sistemas asistenciales se conformaron como diques para el avance del capitalismo industrial. Los reformadores de 1834 interpretaron de esta manera a la ley de Speenhamland, con su reforzamiento de las relaciones paternales en las comunidades rurales, a partir del reconocimiento de un "derecho a vivir" y la condición del apego a la propia parroquia. Con la derogación de esta ley se abrirá una nueva historia en la vinculación entre políticas de asistencia y trabajo.

La nueva ley de pobres expresó las exigencias de un capitalismo industrial que ya tenía como horizonte el mercado mundial. En este sentido, se orientó hacia la creación de un mercado nacional de trabajo libre, y lo hizo en dos sentidos: por un lado con la erradicación de las ataduras sociales y territoriales que fomentaban las leyes anteriores - imponiendo la libertad de circulación de los trabajadores; pero por otro lado y sumado a lo anterior, generando, desde una ahora sí clara distinción entre el pobre "valido" y el incapacitado, la necesidad del trabajo asalariado para aquellos pobres que "sólo poseen la fuerza de sus brazos". Es decir, un proceso tendiente hacia una creciente "mercantilización" de las relaciones laborales. Bajo el principio de "menos elegibilidad", estas nuevas leyes establecieron como único destino para los indigentes sanos el reformatorio, convertido en un espacio denigrante y estigmatizante; no se otorgaron más subsidios francos y la dirección del sistema fue nacional y diferenciada. Con esta transformación, según Polanyi (1992), se produce el nacimiento conjunto de la economía de mercado y de la clase trabajadora moderna.

Esta producción de una línea divisoria en el seno de lo que fue visto de manera retrospectiva como "ambigüedad" en la concepción de la pobreza, forma parte también de un proceso histórico amplio caro a nuestra modernidad: la creciente escisión entre las nociones de pobreza y trabajo. Si en las formaciones sociales preindustriales el trabajo y la pobreza se presentan como una entidad indiferenciada, en el sentido de ésta última como la condición de vida de las clases trabajadoras, con el creciente énfasis en la diferenciación entre pobre válido e inválido que se condensa en las nuevas leyes de pobres del siglo 19, el trabajo y la pobreza vivieron un proceso de separación que implicó no sólo una distinción, sino más bien una oposición (MORELL, 2002). Así, la pobreza del siglo 19, ese pauperismo de masas, se 
convertirá en "lo otro" del trabajo, como consecuencia del incumplimiento de la obligación del mismo y se construirá como pobreza "peligrosa": como condensación de todas las representaciones amenazantes sobre estos grupos pauperizados, generando intensos procesos de criminalización. En realidad, será el mismo proletariado de la ciudad sobre el que recaerá este cúmulo de representaciones asociadas a la peligrosidad durante el siglo 19, pero el hecho de que su trabajo no se encontrara inscripto dentro de un marco regulatorio que le otorgara reconocimiento social, abre la posibilidad a la serie clases laboriosas - clases peligrosas (CASTEL, 2004). El pauperismo será construido como amenaza y progresivamente como lo otro a la obligación al trabajo, pero a su vez producido sobre los cuerpos de los mismos trabajadores privados del reconocimiento social de su trabajo, construida sobre esa "miseria que trabaja" (CASTEL, 1997, p. 69).

Desde el final de siglo 19, pero fundamentalmente en la primera parte del siglo 20, progresivamente el trabajo se constituye como el soporte central del reconocimiento social. Al insertarse gradualmente en una trama cada vez más densa de regulación, a partir de los diferentes arreglos institucionales que conformaron los llamados Estados de Bienestar, el trabajo asalariado se configura como el punto de referencia en donde se articulan toda una serie de derechos que otorgan una identidad, aquella que se funda en el reconocimiento de la utilidad de lo hecho para otros mediante el trabajo. En este proceso, se desdibuja el binomio clases laboriosas/clases peligrosas, y el estatus del trabajo asalariado alcanza a construir verdaderas sociedades salariales (CASTEL, 2004).

Sin embargo, desde mediados de los años setenta y principios de los ochenta asistimos a un nuevo ciclo histórico en nuestras sociedades occidentales. En términos de Fitoussi y Rosanvallon (1996), lo sucedido puede entenderse como crisis de civilización y crisis de individuo; estas comprenden la crisis del Estado providencia como crisis de las instituciones que hacían funcionar el vínculo social y la solidaridad; la crisis del trabajo, a partir de fallas en las instituciones que regulan la relación entre la economía y la sociedad; y por último, ligada a los modos de constitución de identidades individuales y colectivas, una crisis del sujeto. Se debilita fuertemente la condición salarial, no solo en términos del tipo de relación en el mercado de trabajo, sino también abarcando todo el conjunto de bienes y servicios que Castel (2004) agrupó como de propiedad colectiva, con los cuales se conformaba la ciudadanía social que caracterizaba a los estados de bienestar; esto por supuesto implica una redefinición radical de los roles y las capacidades del Estado. Desatada esta dinámica, se observan profundos procesos de heterogeneización de sectores fuertemente integrados en el pasado, a partir de una desafiliación de numerosos individuos que no encuentran fácilmente formas de regenerar lazos de sociabilidad. Situación que ha sido numerosas veces calificada como de "crisis de cohesión".

En el contexto de dichas transformaciones es en el que Castel (2004) introduce la noción del retorno de las clases peligrosas. Consideramos que en esta nueva situación se reactualiza como nueva construcción político cultural, la serie pobreza, peligrosidad y delito. Este retorno de las clases peligrosas como noción que en nuestras sociedades articula dichas tres dimensiones, es un elemento de profunda relevancia para analizar los procesos ligados a la construcción de intervenciones estatales sobre la "nueva cuestión social". Así, desde mediados de los años 1970, y con mayor fuerza durante los años 1980, comienza a cristalizarse una intensa reorientación de las lógicas estatales de intervención. Este proceso tiene una clara expresión tanto en el campo de la política social como en el ámbito de la política criminal. Las políticas sociales, como instancia estatal de constitución de la cuestión social, reflejaron este cambio en el reposicionamiento de las estrategias asistenciales, tanto en una mayor presencia de las mismas dentro del campo de la política social, como en la mutación de las concepciones generales que lo organizaron. Proceso que se conjugó con una centralización de la noción de pobreza (GRASSI; HINTZE; NEUFELD, 1994, GRASSI, 2003; DANANI, 1999, ALVAREZ LEGUIZAMÓN, 2005). En cuanto a la política criminal, en esos años se observa una verdadera crisis de las estructuras institucionales que se habían consolidado durante todo el siglo 20 en este campo. La desarticulación del complejo penal-welfare (GARLAND, 2005) tuvo una de sus expresiones fundamentales en la aparición de la noción de nueva prevención: la proliferación de formas de prevención del delito que 
desbordan las intervenciones exclusivamente penales (BARATTA, 1997; SOZZO, 2000).

Planteados estos elementos, consideramos que emerge la necesidad de analizar dichas reorientaciones en los campos de la política social (ligada a las nociones de pobreza y condiciones de vida) y de la política criminal (centrada en la definición de delincuente) en forma conjunta, relacional, preguntándonos por la naturaleza de su vinculación en los procesos históricos de construcción o "reactualización" de esa categoría que jugó (y juega, según entendemos) un papel fundamental en la constitución del orden social: las clases peligrosas. Dadas las transformaciones que apuntamos, el entrecruzamiento entre las intervenciones asistenciales y las estrategias de prevención social del delito se presenta como un lugar privilegiado para el estudio de dichos procesos, en tanto que configuran espacios de intersección entre los campos de la política social y la política criminal. Resultan de especial importancia las definiciones de la pobreza y lo delictivo que se construyen en dichas políticas, particularmente en los mecanismos de delimitación de su "población objetivo" (DANANI, 1996).

\section{Resultados}

Los caminos de la prevención: descentramiento de la intervención en las condiciones de vida e individualización

Ahora presentaremos los elementos emergentes que refieren a lo que llamamos "mecanismos preventivos".

Una primera cuestión a tratar es la construcción de una noción de "programa", que aparece tanto en las entrevistas a operadores como en documentos de gestión del mismo, y que considera a la intervención de prevención social como una entidad diferenciada de la intervención que, en una misma población-objetivo, implica el otorgamiento de PEC. En otras palabras, aunque desde el punto de vista del funcionamiento, el PCV y el PEC se presentan integrados como intervención sobre una población-objetivo que "recortan" de manera mutua, la construcción del "programa" de prevención del delito surge como un espacio diferenciado del PEC. La prevención del delito y la puesta en marcha de sus mecanismos preventivos específicos suceden en un campo más allá del PEC, y en algún sentido luego de él. Dentro de esta diferenciación el PEC aparece como una "herramienta" externa, que permite y mantiene el ingreso de los destinatarios dentro del "programa", dentro del "verdadero" espacio de prevención del delito.

Entonces, para nosotros siempre lo que tuvimos en claro es que era una herramienta [...]
La idea es que sirva como una herramienta para acceder a otros aspectos de la vida de ese joven que nos preocupa y creemos que tenemos que trabajar más en ese punto [...] (Entrevista n. 8 Autoridad Local).

A ver, a nosotros nos sirvió como la manzana, para atraerlos; como la zanahoria, digamos. Como decía una compañera mía, es la zanahoria para atraerlos y poder trabajar (Entrevista n. 5 - Operadora).

Consideramos que este punto es de suma importancia puesto hace al centro de la relación entre intervenciones preventivas y políticas sociales. Al entender a la intervención de política social sólo como herramienta para insertar a la población objetivo al interior del dispositivo de prevención, se produce un descentramiento de los mecanismos preventivos ligados al impacto en las condiciones de vida de la población destinataria. Esto es de gran relevancia para caracterizar a una intervención que se inscribe explícitamente dentro de la prevención social del delito.

\section{La intervención sobre una individualidad}

Dicho espacio, que se configura como el lugar propio de la estrategia de prevención del delito, es construido como un espacio de intervención sobre la subjetividad, sobre la individualidad, que se presenta en la figura del "jóvenes en conflicto con la ley penal". Las operaciones que se establecen en el PCV como preventivas del delito se vinculan a una mecánica de transformación sobre las individualidades que conforman la "población-objetivo". Este trabajo es descrito como una tarea minuciosa, continua, ardua, y fundamentalmente a largo plazo: es un trabajo "de hormiga".

[...] entonces por más que venga por el PEC vos lo tenías tendido ahí para laburarlo y vos tenias que aprovechar esa oportunidad para laburarlo, era lo que vos tenías para agarrarlo y para que venga una vez por semana, dos veces por semana, para moldearlo, para tratar de laburar con el pibe [...] (Entrevista n. 6-Operadora).

La idea de "laburar" al pibe, de trabajar sobre su subjetividad es uno de los mecanismos preventivos fundamentales que hemos identificado en nuestro análisis. Este funcionamiento recorre transversalmente los tipos de intervención que hemos descripto, tanto los talleres, las reuniones grupales como las entrevistas individuales. Podemos diferenciar dos formas fundamentales del mismo, el encuadre, y el proyecto de vida.

El encuadre es un trabajo sobre la individualidad que aparece fuertemente ligado a la noción de límite: 
la intervención se presenta como sumamente vinculada a esta producción. Como respuesta a una subjetividad que es caracterizada por la dificultad de aceptar reglas de conducta, el marcar límites y establecer normas será interpretado como uno de las estrategias más productivas en esta intervención sobre los destinatarios. Y la misma desbordará los dispositivos formales establecidos por el PCV, como ser las reuniones grupales y las entrevistas individuales, para impregnar el conjunto de las interacciones entre el operador y el destinatario. Así, aparece como propiedad común en los documentos y entrevistas analizadas, que producir respeto por los horarios de las entrevistas, cuidado para la utilización de la palabra en las reuniones grupales y observancia de todas las pautas de interacción acordadas, se constituían en uno de los principales mecanismos de prevención. Esta idea de producción de límites, entendida como la instauración en el ámbito de lo subjetivo de las normas básicas que permiten la interacción, es condensada bajo la noción de encuadre.

Por lo general, por mi experiencia acá en este programa, ellos son jóvenes que nunca han tenido límites, de familia, de escuela [...]. Yo creo que ya desde marcarle un encuadre en el programa, tener que respetar un horario de entrevista, o de grupo, tener que quedarse ahí sentado una hora en el grupo sin tener que irse; es como que van marcando de a poco unas ciertas normas que por ahí él no vivió en su casa (Entrevista n. 4 - Operadora).

Las referencias a la familia, a la casa y al colegio no son casuales. Circula dentro de esta forma de intervención sobre los destinatarios del programa una asimilación a los procesos ligados a la educación de los niños, tanto en el ámbito de la familia, como en el de la escuela.

Viste ese 'parate' que uno dice, no, 'hoy voy a hacer esto y mi viejo me mata, si hago esto mi viejo me mata'. Ellos tienen falla en esto porque no hubo ahí adultos que instalen eso, eso lo venimos a querer instalar nosotros diez, quince, veinte años después lo que tenía que haberse instalado (Entrevista n. 3 - Operadora).

Lo que aparece es una construcción que presenta un diagnóstico sobre la falla subjetiva en torno a la incorporación de normas en los procesos que podrían entenderse como de socialización "primaria", en virtud de un mal funcionamiento de las instituciones organizadoras de este proceso, como la familia y la escuela. A partir del mismo es que se presenta el trabajo de "encuadre" como un núcleo fundamental en este proceso de transformación individual. Este pasaje de un diario de operador expresa dicha dinámica a partir de la asimilación del trabajo de operador con el rol de madre:

[...] y ahí Dora dijo que ella ya le había dicho que se tenía que componer sino iba a jugar con pañales, ahí él reaccionó re-mal, le dijo 'callate la boca que te arranco la cabeza', yo le dije 'no le hables así a tu mamá' y a continuación le bromeé que su madre y yo juntas lo teníamos loco (Diario de Operador n. 1).

El sentido de estas operaciones sobre la individualidad también se vincula a la creación de un espacio de comunicación, especialmente en la relación operador-destinatario dentro de las entrevistas individuales, que es presentado fundamentalmente como diferenciado y en contraposición de los espacios sociales "naturales" de los destinatarios. Este otro espacio aparece como propicio para expresarse y escuchar, para generar un ámbito de reflexión sobre las propias prácticas y sus consecuencias. A partir principalmente de "aportar otra mirada", proponer algo distinto a lo que instan sus ámbitos cotidianos.

[...] porque los pibes lo que necesitan no es que los escuche alguien como los escucha el amigo de la esquina, o el vecino o el familiar que por ahí le va a decir más de lo mismo... El tema es que lo que él diga no caiga en saco roto, en el sentido que él pueda recibir de eso que está contando una respuesta que a lo mejor a él no se le ... algo que él no ve, o que a él no se la había ocurrido, que por ahí intuye pero no puede terminar de cerrar (Entrevista n. 3 - Operadora).

Estas intervenciones que marcan límites y se presentan como aportando un espacio de comunicación diferente, son reconstruidas como las generadoras de lo que hemos conceptualizado como las "precondiciones de un proceso de inclusión social". Decimos precondiciones porque aparecen con el objetivo de producir que la inserción en nuevas dinámicas sociales sea percibida como posibilidad. Constituir como posibilidad cursos de vida diferente es una de las metas principales con las que se presentan estas operaciones sobre la individualidad.

[...] sobre todo con los pibes que ya habían pasado por un primer proceso, que ya habían hecho los talleres, que ya se había trabajado bastante con la subjetividad y demás, estaban en mejores condiciones de poder aceptar eso como posibilidades, como oportunidades, y así y todo, necesitaban un cuerpo a cuerpo para darle continuidad, recibirse [...] (Entrevista n. 2 Autoridad Nacional). 
[...] la prevención que hacíamos nosotros, era el paso anterior, lo que hacíamos nosotros, para que el pibe pueda insertarse en un taller o para que el pibe pueda leer y estudiar algo o aprender un oficio, era anterior a eso, ni siquiera el PEC como plan de empleo y capacitación... o sea era antes, porque si no, si lo mandabas a una capacitación pero el pibe dejaba a los dos días, porque dejan todo, porque no tienen interés [...] (Entrevista n. 6-Operadora).

Como vemos, el trabajo con estos jóvenes se presenta en ambos extractos como el paso necesariamente anterior, de otros procesos que son entendidos por los agentes del PCV en términos de "reinserción social". Y se constituye como necesariamente anterior dado que es una instancia habilitante, que otorga al "joven" de las capacidades necesarias para jugar ese juego. En el caso el primer extracto se refiere a experiencias de trabajo auspiciadas por el programa; el segundo es más general y se refiere a otras instancias de capacitación. Pero en ambos la posibilidad de "reinserción" en estos espacios tiene como condición un trabajo previo sobre la subjetividad de los destinatarios

El proyecto de vida sugiere el sujeto activo, que surge de la construcción acerca de constituir como posibilidad cursos de vida diferentes. En nuestros referentes empíricos, encontramos que uno de los objetivos explícitos de los espacios de intervención que el programa pone en juego es el de generar en los destinatarios la capacidad de orientarse a partir de esta noción. Por supuesto, esta idea de habilitar espacios que permitan lo que en su diagnóstico aparece como obturado en los jóvenes, supone una oposición al curso de vida por el cual ellos y ellas transitaron y transitan. En uno de los documentos analizados, un diario de operador, esta oposición se expresa en términos extremos de una pugna entre un proyecto de vida, que intenta generar el programa, y un proyecto de "muerte", entendiendo por este una vida ligada al delito.

Estamos intentando que mantengan un proyecto de vida no un proyecto de muerte, estar preso es una muerte social. El PCV pretende junto con ustedes que puedan armarse cada uno en un grupo un proyecto de vida que no los lleve a la muerte (Diario de Operador n. 2).

La forma más cristalizada institucionalmente que expresa esta orientación es la puesta en práctica de un taller organizado desde la coordinación nacional del programa, llamado Taller de Proyecto de Vida e Identidad. En la sistematización del taller, observamos que el mismo se organiza en diez encuentros, estructurados en tres módulos. Las dinámicas que propone para realizar los objetivos del mismo están ligadas a actividades prácticas, como el dibujo, el collage, la elaboración de escritos, trabajando individualmente y en plenario. Esta intervención se caracteriza fundamentalmente por orientarse hacia la generación de capacidades individuales que permitan la formación de un proyecto de vida. Entre las cuales se cuenta:

Desarrollar la capacidad de reconocer puntos de vista diferentes [...]. [...] la capacidad de analizar los sucesos en una lógica de causa - efecto concatenados con otros, fomentando así el desarrollo del 'locus de control interno' [...] [Desarrollar la capacidad para] [...] la reconstrucción de historias individuales, con miras a elaborar la ubicación temporal-espacial y ubicar diversos factores actuantes en el desarrollo y actualidad de la vida de una persona, así como en la construcción de condiciones y posibilidades de proyección a futuro (Sistematización Taller de Proyecto de Vida e Identidad).

Como podemos observar, la noción del "desarrollo de capacidades", hace pié en un terreno fundamentalmente individual, mediante un registro fuertemente psicológico. Se presenta como una operación dispuesta a lograr una activación subjetiva que, a partir de un aumento en su capacidad reflexiva, pueda comprender su pasado y sobre todo, controlar su futuro. La idea de desarrollar la capacidad para la estructuración de un proyecto de vida se articula en torno a una construcción que se orienta hacia un aumento en la facultad de dominio sobre los cursos de vida adoptados, y suponiendo una población-objetivo que se caracterizaría justamente por su incapacidad al respecto. Por ello, dicha transformación individual se construye como una generación de una actitud fundamentalmente "activa". Los espacios de intervención establecidos por el PCV son presentados como instando a un "desarrollo activo de capacidades individuales" que permitan el cambio, pero teniendo siempre como condición esa activación subjetiva.

Hubo cambios personales, hubo mucha revinculación familiar. O sea, hubo cambios, para mi forma de ver, extraordinarios. No digo que el programa sea el artífice porque el artífice es el pibe que lo busca; pero sí que justo él quería cambiar y sí, se cruzó con este programa y ahí surgió algo; eso sí (Entrevista n. 3-Operadora).

Surge la noción de que el programa debe articularse con una actitud activa del destinatario orientada al cambio, y correr por ese camino. Y este mecanismo se posa también sobre uno de los temas fundamentales acerca de las construcciones de sen- 
tido en torno a la población delimitada para la intervención: la relación con el mundo del trabajo. La reconstrucción de un posicionamiento "activo" se expresará significativamente en la cuestión de la "búsqueda" de trabajo.

\section{Discusión}

El PCV estableció una relación compleja con el ámbito de la política social. Aunque por un lado se construye la "naturalidad" de la confluencia entre las estrategias de prevención del delito e intervenciones sociales dado su carácter anticipatorio, es decir, su enfoque no en la comisión del delito sino en los procesos o "causas" sociales que lo harían surgir, las relaciones concretas que se articularon hablan, en los distintos niveles en los que lo analizamos, de una cierta "exterioridad" de la política social con respecto a los procesos que intentaban generar prevención del delito.

El núcleo de este proceso lo desarrollamos en la distinción entre el "verdadero" espacio de intervención y el PEC. A su vez, esta forma de estructurar los mecanismos preventivos tiene central importancia porque supone una tendencia hacia la descentralización del impacto en las condiciones de vida, mecanismo "natural" de las intervenciones de prevención "social” del delito. Es decir, se desdibuja el papel de la política social, y su impacto en las condiciones de vida, como mecanismo preventivo organizador de la intervención. Y esto es fundamental para una política que las construía como de central importancia para actuar sobre las "causas", o los "orígenes" de los comportamientos delictivos, imprescindible para una intervención que organizó su proceso de delimitación de los territorios y las poblaciones a intervenir a partir de preguntarse por su contexto. Esta orientación estructuró a la intervención hacia el campo de las intervenciones cuyo objeto son las condiciones de vida, y en esa relación construyó qué era lo "social" para esta estrategia de prevención social del delito.

Consideramos que esta orientación que adquiriera la confluencia del PCV y el PEC no puede entenderse sin observar algunas características que presenta el campo de las intervenciones sociales. Nos referimos en primer lugar a los procesos que fueron conceptualizados a partir de la noción de "indi"vidualización” de la protección (DANANI, 2005). La misma es caracterizada como

Una forma específica de la orientación de la protección social que se caracteriza por atar las condiciones de vida de las personas a la situación y las prácticas en sentido amplio, del sujeto en tanto individuo; y que, a la vez desvincula esas condiciones de vida y las consecuencias de esas prácticas de toda relación con y de toda pertenencia a grupos o agregados mayores (DANANI, 2005, p. 2).

Entendemos que aquí emerge una productiva clave interpretativa: en el sentido de hasta qué punto la estructuración del campo de la política social a partir de estos procesos es una de las condiciones fundamentales para entender la orientación que hemos descrito en relación a las intervenciones propuestas por el PCV y los mecanismos preventivos que estas intentaban poner en funcionamiento. Al orientarse esta estrategia de prevención del delito hacia el ámbito de lo social, conjugando al PCV con el PEC, las dinámicas descriptas en el interior de dicho campo podrían haber condicionado sus lógicas. Es decir, la estructura de este campo podría explicarnos de manera satisfactoria los procesos de individualización que presenta la articulación del PCV y el PEC. Creo que el descentramiento de las condiciones de vida y la centralización del individuo como espacio de intervención pueden entenderse en este sentido. Y también mostrando una reactualización de la serie pobreza-peligrosidad-delito fuertemente individualizada.

\section{Referencias}

ALVAREZ LEGUIZAMÓN, S. Introducción. En: (Comp.). Trabajo y producción de la pobreza en Latinoamérica y el Caribe: estructuras, discursos y actores. Buenos Aires: Clacso, 2005, p. 19-53.

BARATTA, A. Política Criminal: entre la política de seguridad y la política social. En: CARRANZA, E. (Coord.). Delito y seguridad de los habitantes. México DF: Siglo XXI, 1997, p. 80-98.

CASTEL, R. Las metamorfosis de la cuestión social. Una crónica del salariado. Buenos Aires: Paidós, 1997.

La inseguridad social. ¿Qué es estar protegido? Buenos Aires: Manantial, 2004.

DANANI, C. Algunas precisiones sobre la política social como campo de estudio y la noción de población-objeto. En: HINTZE, S. (Org.). Políticas sociales. Contribución al debate teórico-metodológico. Buenos Aires: CBC-UBA, 1996, p. 21-38.

De la heterogeneidad de la pobreza a la heterogeneidad de los pobres. Comentarios sobre la investigación social y las políticas sociales. Revista Sociedad, Buenos Aires, UBA, n. 14, p. 29-41, ago. 1999.

Las políticas sociales de los '90: los resultados de la combinación de individualización y comunitarización de la protección. Disponible en: http://www.tau.org.ar/ 
upload/89f0c 2 b6 65 ca 02 ff 45 ef6 1 a 4 f 2 e 5 bf $24 /$ Comunitarizaci_n_e_individualizaci_n_Claudia_Danani.pdf. Acceso en: fev. 2010.

FITOUSSI, J. P.; ROSANVALLON, P. La era de las desigualdades. Buenos Aires: Manantial, 1996.

GARLAND, D. La cultura del control. Crimen y orden social en la sociedad contemporánea. Barcelona: Editorial Gedisa, 2005.

GLASER, B.; STRAUSS, A. The Discovery of Grounded Theory. Nueva York: Aldine Publishing Company, 1967.

GRASSI, E.; HINTZE, S.; NEUFELD, M. et al. Politicas sociales, crisis y ajuste estructural. Buenos Aires: Espacio Editorial, 1994.

GRASSI, E. Política y problemas sociales en la sociedad neoliberal. La otra década infame. Buenos Aires: Espacio Editorial, 2003.

HIMMELFARB, G. La idea de la pobreza. Inglaterra a principios de la era industrial. México: Fondo de Cultura Económica, 1983.

MORELL, A. La legitimación social de la pobreza. Madrid: Editorial Anthropos, 2002.

POLANYI, K. La gran transformación. Los orígenes políticos y económicos de nuestros tiempos. México: Fondo de Cultura Económica, 1992.

SONEIRA, J. 'La teoría fundada en los datos', (Grounded Theory) de Glaser y Strauss. En: JORNADAS DE ETNOGRAFÍA Y MÉTODOS CUALITATIVOS 4. Buenos Aires: IDES, 2004.

SOZZO, M. Seguridad urbana y técnicas de prevención del delito. Cuadernos de Jurisprudencia y Doctrina Penal. Buenos Aires, n. 10, p. 17-82, 2000.

\section{Nota}

1 El Plan Jefas y Jefes de Hogar Desocupados estuvo destinado a jefas y jefes de hogar en situación de desempleo que tuvieran a cargo menores de edad o personas discapacitadas.

\section{Emilio J. Ayos}

emilio.ayos@gmail.com

Doctorando en la Facultad de Ciencias Sociales de la Universidad de Buenos Aires (FSOC-UBA)

Direción: Prof. Dra. Claudia Danani

Docente en la Carrera de Trabajo Social (FSOCUBA)
FSOC - UBA

Instituto de Investigaciones Gino Germani Facultad de Ciencias Sociales (UBA)

Pte. J. E. Uriburu $9506^{\circ}$

1114

Buenos Aires - Argentina 